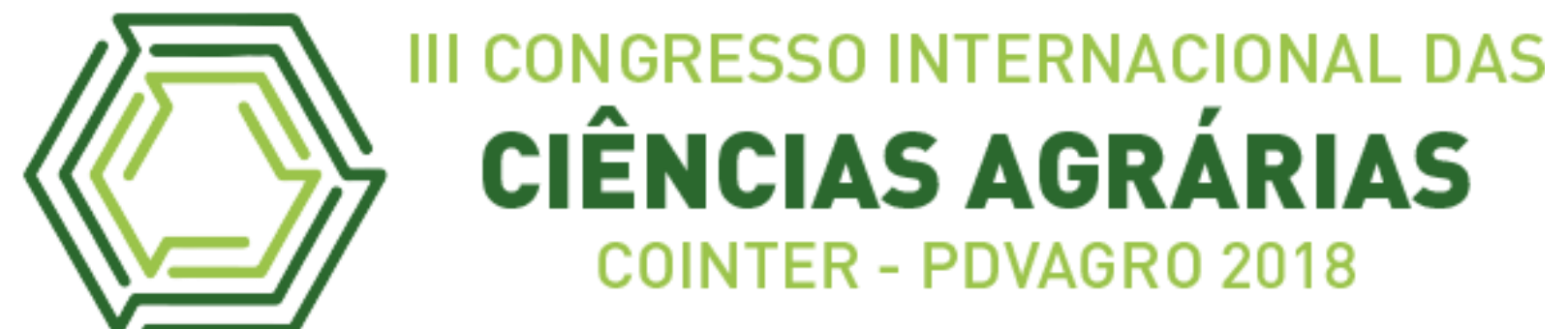

\title{
CARACTERIZAÇÃO DE ÓXIDOS DE FERRO E AGREGAÇÃO EM CLASSES DE SOLOS NA CHAPADA DO APODI
}

\section{CHARACTERIZATION OF IRONS AND AGGREGATION IN SOIL CLASSES IN CHAPADA APODI}

Apresentação: Pôster

Valéria Nayara Silva Oliveira ${ }^{1}$; Jeane Cruz Portela ${ }^{2}$; Thais Cristina de Souza Lopes ${ }^{3}$; Kellyane da Rocha Mendes ${ }^{4}$; Clarisse Pereira Benedito ${ }^{5}$

\section{DOI: https://doi.org/10.31692/2526-7701.IIICOINTERPDVAGRO.2018.00362}

\section{Introdução}

A agregação regula atributos como distribuição de poros por tamanho, aeração, dinâmica da água, penetração de raízes, resistência à ação dos agentes ativos (água, vento), agindo diretamente na superfície do solo, pois a agregação refere-se a união das partículas inorgânicas e orgânicas, e a sua interação com os agentes cimentantes como os óxidos de ferro, os carbonatos, o carbono orgânico total e outros, que refletirá em condições diferenciadas, quanto a união das mesmas. O grau de cristalinidade dos óxidos tem sido comumente estudado pela relação entre os teores do elemento Fe extraído com oxalato ácido de amônio e com DCB, indicando a reserva do ferro em função de outros grupos minerais, ferro amorfo e cristalino (Feo/Fed) (KÄMPF, 1988). Essa avaliação é utilizada para avaliar o grau da pedogênese nas classes de solos.

Assim, este trabalho teve como objetivo caracterizar a agregação e suas inter-relações entre as formas extraíveis de óxidos de ferro, como também, carbono orgânico total e a fração ativa do solo (argila), em classes de solos.

\footnotetext{
${ }^{1}$ Mestranda em Ambiente, Tecnologia e Sociedade, Universidade Federal Rural do Semi-árido - UFERSA, valeria-nayara@hotmail.com

${ }^{2}$ Professora da Universiade Federal Rural do Semi-Árido - UFERSA, jeaneportela@ufersa.edu.br

${ }^{3}$ Doutora do Programa de Pós- Graduação em Manejo de Solo e Água - Universidade Federal Rural do Semiárido - UFERSA, thaiscristina13@hotmail.com

${ }^{4}$ Mestranda em Manejo de Solo e Água, Universidade Federal Rural do Semi-árido - UFERSA kellyane.mendes@hotmail.com

${ }^{5}$ Professora da Universidade Federal Rural do Semi-árido - UFERSA, clarisse@ufersa.edu.br
} 


\section{Metodologia}

A pesquisa foi realizada no Projeto de Assentamento Moacir Lucena, município de Apodi, no Rio Grande do Norte na região Semiárida do Brasil. Foram selecionados sete ambientes, sendo definidos pelos perfis apresentados na tabela 1.

\section{Fundamentação Teórica}

Os teores de (Fed) variam em função da litologia, de acordo com Pötter \& Kämpf (1981) e Kämpf \& Dick (1984), rochas ácidas com o material de origem arenito, oriundo das rochas sedimentares, apresentam valores inferiores de Fed que as rochas básicas. Diante do exposto, as classes de solos em estudo apresentam forças de coesão e adesão diferentemente, em função dos fatores e processos de formação, como também, na sua agregação.

Tabela 1. Usos, classificação e localização dos ambientes estudados. . Fonte: Própria

\begin{tabular}{|c|c|c|c|c|}
\hline Perfil & Horizonte & Classe & Usos & Localização \\
\hline 1 & Bt $(48-120)$ & $\begin{array}{c}\text { Latossolo Vermelho- } \\
\text { Amarelo }\end{array}$ & $\begin{array}{c}\text { Área em } \\
\text { recuperação }\end{array}$ & $\begin{array}{l}05^{\circ} 32^{\prime} 05,2^{\prime \prime ~ S} \\
37^{\circ} 52^{\prime} 55,7^{\prime \prime} \mathrm{W}\end{array}$ \\
\hline 2 & $\mathrm{Bi}(15-54)$ & Cambissolo Háplico & Área da lagoa & $\begin{array}{l}05^{\circ} 32^{\prime} 17,9^{\prime \prime} \mathrm{S} \\
37^{\circ} 53^{\prime} 09,9^{\prime \prime} \mathrm{W}\end{array}$ \\
\hline 3 & Bt (25 - 97) & Latossolo Amarelo & $\begin{array}{l}\text { Área de } \\
\text { reserva }\end{array}$ & $\begin{array}{c}05^{\circ} 32^{\prime} 09,8^{\prime \prime} \mathrm{S} \\
37^{\circ} 53^{\prime} 18,1^{\prime \prime} \mathrm{W}\end{array}$ \\
\hline 4 & $\mathrm{Bw}(16-95)$ & Argissolo Amarelo & $\begin{array}{c}\text { Área } \\
\text { agroecológica }\end{array}$ & $\begin{array}{l}05^{\circ} 33^{\prime} 16,8^{\prime \prime} \mathrm{S} \\
37^{\circ} 53^{\prime} 19,1^{\prime \prime} \mathrm{W}\end{array}$ \\
\hline 5 & $\mathrm{Bi}(18-60)$ & Cambissolo Háplico & Área coletiva & $\begin{array}{l}05^{\circ} 32^{\prime} 07,9^{\prime \prime} \mathrm{S} \\
37^{\circ} 53^{\prime} 51,6^{\prime \prime} \mathrm{W}\end{array}$ \\
\hline 6 & Bt $(40-120)$ & Latossolo Amarelo & $\begin{array}{l}\text { Área do } \\
\text { cajueiro }\end{array}$ & $\begin{array}{c}05^{\circ} 31^{\prime} 53,7^{\prime \prime} \mathrm{S} \\
37^{\circ} 53 ' 50,8^{\prime \prime} \mathrm{W}\end{array}$ \\
\hline 7 & $\mathrm{Bt}(23-75)$ & Latossolo Amarelo & $\begin{array}{l}\text { Área do } \\
\text { cajueiro }\end{array}$ & $\begin{array}{l}05^{\circ} 31^{\prime} 51,2^{\prime \prime} \mathrm{S} \\
37^{\circ} 54^{\prime} 02,9^{\prime \prime} \mathrm{W}\end{array}$ \\
\hline
\end{tabular}

Para caracterização dos ambientes coletou-se amostras com estruturas deformadas e indeformadas nas áreas supracitadas, nos horizontes diagnósticos. As amostras deformadas foram acondicionadas em sacos plásticos e seu beneficiamento em terra fina seca ao ar (TFSA). Os atributos químicos e físicos estudados foram: carbono orgânico total (COT), cálcio $\left(\mathrm{Ca}^{2+}\right)$ e sódio $\left(\mathrm{Na}^{+}\right)$e a fração argila $(<0,002 \mathrm{~mm})$. E as amostras indeformadas foram coletadas em blocos para as análises de estabilidade de agregados: diâmetro médio ponderado (DMP) e diâmetro médio geométrico (DMG), e os atributos estruturais do solo em anéis volumétricos: macroporosidade (Ma), microporosidade (Mi), porosidade determinada (Ptdet), porosidade de aeração (Paeração). Analisadas no complexo do Laboratório de Análises de Solo, Água e Planta do Centro de Ciências Agrárias da Universidade Federal Rural do Semiárido (LASAP-CCA-UFERSA), seguindo a metodologia proposta por TEIXEIRA et al. (2017). 
Foram realizadas três análises distintas para quantificação dos óxidos de ferro presentes nos horizontes diagnósticos dos solos estudados. Inicialmente foi realizado o ataque sulfúrico (VETTORI, 1969), e quantificado o ferro presente nos minerais secundários (Fes), na fração argila. A determinação de Fe foi realizada por Espectrofotometria de Absorção Atômica (TEIXEIRA et al.2017).

Em seguida foi realizada a determinação do ferro por DCB (Ditionito-CitratoBicarbonato), para quantificação do Fe em formas cristalinas (Fed) (MEHRA \& JACKSON, 1960). Posteriormente foi realizada a determinação do ferro por oxalato, para quantificação do ferro em formas amorfas (Feo) (MEHRA \&JACKSON, 1960).

Para interpretação dos atributos mais sensíveis na distinção dos ambientes em estudo, os dados foram submetidos à análise de estatística multivariada como ferramenta principal, especificamente por meio das análises de componentes principais, fatorial e agrupamento (Statistica 7, 2004).

Foi utilizada a análise de correlação de Pearson $(\mathrm{p} \leq 0,05)$ para as 15 variáveis com o objetivo de garantir que estas possuíssem correlações mínimas suficientes para justificar o seu uso na matriz de dados na $\mathrm{AF}$. Para a $\mathrm{AF}$, os fatores com autovalores maiores que 1 foram extraídos por componentes principais, e os eixos fatoriais foram rotacionados pelo método Varimax. Para este estudo estabeleceu-se o valor de 0,65 para cargas fatoriais significativas (CASTILHO, 2017).

\section{Resultados e Discussões}

Correlações significativas foram encontradas entre Argila e $\mathrm{Na}^{+}$, assim como o DMP com Fes, Fed e porosidade de aeração. As variáveis Fes, Feo/Fed, Ptd, DMG, Ma, $\mathrm{Na}^{+}, \mathrm{Ca}^{2+}$, Dse COT não apresentaram correlação com as demais, podendo afirmar que estas são independentes (Tabela 2).

Tabela 2 - Matriz de correlação entre as variáveis dos atributos do solo nas áreas em estudo, no Projeto de Assentamento Terra da Esperança - RN. . Fonte: Própria

\begin{tabular}{|c|c|c|c|c|c|c|c|c|c|c|c|c|c|c|c|}
\hline & Argila & Fes & Fed & $\mathrm{Feo}$ & $\mathrm{Feo} / \mathrm{Fed}$ & Ptd & $\mathrm{Pa}$ & DMP & DMG & Mi & $\mathrm{Ma}$ & $\mathrm{Na}^{+}$ & $\mathrm{Ca}^{2+}$ & Ds & COT \\
\hline Argila & 1,00 & & & & & & & & & & & & & & \\
\hline Fes & $-0,30$ & 1,00 & & & & & & & & & & & & & \\
\hline Fed & 0,16 & 0,29 & 1,00 & & & & & & & & & & & & \\
\hline Feo & 0,10 & 0,00 & 0,51 & 1,00 & & & & & & & & & & & \\
\hline Feo/Fed & $-0,10$ & $-0,32$ & $-0,52$ & 0,41 & 1,00 & & & & & & & & & & \\
\hline Ptd & $-0,15$ & 0,28 & $-0,01$ & 0,54 & 0,59 & 1,00 & & & & & & & & & \\
\hline $\mathrm{Pa}$ & 0,00 & 0,29 & 0,66 & 0,73 & $-0,03$ & 0,63 & 1,00 & & & & & & & & \\
\hline DMP & 0,13 & $-0,33$ & $-0,82$ & $-0,80$ & 0,01 & $-0,49$ & $-0,84$ & 1,00 & & & & & & & \\
\hline DMG & 0,23 & $-0,14$ & $-0,69$ & $-0,67$ & $-0,02$ & $-0,03$ & $-0,37$ & 0,78 & 1,00 & & & & & & \\
\hline $\mathrm{Mi}$ & $-0,06$ & $-0,48$ & 0,41 & 0,41 & $-0,11$ & $-0,02$ & 0,54 & $-0,40$ & $-0,27$ & 1,00 & & & & & \\
\hline $\mathrm{Ma}$ & 0,03 & 0,00 & $-0,69$ & $-0,58$ & 0,24 & $-0,26$ & $-0,88$ & 0,71 & 0,37 & $-0,85$ & 1,00 & & & & \\
\hline $\mathrm{Na}^{+}$ & 0,84 & $-0,04$ & $-0,04$ & $-0,30$ & $-0,29$ & $-0,42$ & $-0,40$ & 0,44 & 0,36 & $-0,47$ & 0,44 & 1,00 & & & \\
\hline $\mathrm{Ca}^{2+}$ & 0,65 & $-0,38$ & 0,38 & 0,61 & 0,02 & $-0,03$ & 0,44 & $-0,26$ & $-0,17$ & 0,55 & $-0,54$ & 0,27 & 1,00 & & \\
\hline Ds & 0,30 & $-0,48$ & 0,30 & $-0,35$ & $-0,53$ & $-0,74$ & $-0,34$ & 0,15 & $-0,12$ & 0,26 & 0,00 & 0,31 & 0,06 & 1,00 & \\
\hline COT & 0,12 & 0,32 & $-0,07$ & $-0,31$ & 0,03 & 0,15 & $-0,30$ & 0,07 & 0,07 & $-0,75$ & 0,59 & 0,34 & $-0,59$ & 0,10 & 1,00 \\
\hline
\end{tabular}


A tabela 3 apresenta as cargas fatoriais dos atributos dos solos analisados e seus respectivos autovalores, variâncias totais observadas e acumulada, são apresentados os resultados utilizando critérios para determinar a quantidade de fatores suficientes para a análise, onde considera as variáveis que expliquem pelo menos $80 \%$ da variabilidade total dos dados com os autovalores, como também, a explicação das variâncias associadas aos fatores gerados e a explicação das variâncias acumuladas. Obtendo assim, como principais resultados os fatores 1, 2, 3 e 4 os quais explicaram 87,24 \% da variação dos dados, considerando apenas as variáveis em destaque (negrito), conforme apresentado na tabela 3 (VICINI, 2005).

De acordo com a tabela 3 representada pelas cargas fatoriais, podemos concluir que o fator 1, considerado fator de maior influência na diferenciação dos ambientes com a variância total de 37,38, está relacionado as variáveis de agregação (Pa, DMP, DMG, Ma) e os teores de $\mathrm{Fe}(\mathrm{Fed} \mathrm{e} \mathrm{Feo).}$

Tabela 3: Fatores extraídos por componentes principais, destacando os atributos do solo com cargas superiores a 0,65 (módulo), no estudo dos horizontes de Cambissolos em diferentes usos. . Fonte: Própria

\begin{tabular}{c|cccc}
\hline & Fator 1 & Fator 2 & Fator 3 & Fator 4 \\
\hline Argila & 0,02 & $-0,09$ & 0,05 & $\mathbf{0 , 9 9}$ \\
Fes & $-0,39$ & 0,07 & $\mathbf{- 0 , 7 5}$ & $-0,24$ \\
Fed & $\mathbf{- 0 , 9 2}$ & $-0,36$ & $-0,02$ & 0,15 \\
Feo & $\mathbf{- 0 , 7 2}$ & 0,52 & 0,28 & 0,18 \\
Feo/Fed & 0,25 & $\mathbf{0 , 8 5}$ & 0,15 & $-0,02$ \\
Ptd & $-0,33$ & $\mathbf{0 , 8 6}$ & $-0,15$ & $-0,11$ \\
Pa & $\mathbf{- 0 , 8 6}$ & 0,33 & 0,19 & 0,00 \\
DMP & $\mathbf{0 , 9 6}$ & $-0,18$ & 0,01 & 0,10 \\
DMG & $\mathbf{0 , 7 2}$ & 0,07 & $-0,02$ & 0,17 \\
Mi & $-0,43$ & $-0,14$ & $\mathbf{0 , 8 6}$ & $-0,13$ \\
Ma & $\mathbf{0 , 7 8}$ & 0,01 & $-0,53$ & 0,07 \\
Na+ & 0,29 & $-0,30$ & $-0,31$ & $\mathbf{0 , 8 4}$ \\
Ca2+ & $-0,36$ & 0,09 & 0,60 & $\mathbf{0 , 6 7}$ \\
Ds & 0,06 & $\mathbf{- 0 , 8 5}$ & 0,19 & 0,19 \\
COT & 0,15 & $-0,01$ & $\mathbf{- 0 , 8 4}$ & 0,12 \\
\hline Expl.Var & 4,91 & 2,86 & 2,95 & 2,37 \\
Variância Total $(\%)$ & 37,38 & 21,44 & 15,18 & 13,24 \\
Variância Acumulada & 37,38 & 58,82 & 74,00 & 87,24 \\
(\%) & & & &
\end{tabular}

O fator 2 em destaque (Tabela 3, Figura 1 e 2) é representado pela relação Feo/Fed, Ptd e Ds. Quantos aos demais fatores (fator 3 e fator 4) estes foram menos expressivos, com valores de cargas fatoriais inferiores, para os atributos selecionados, as quais se destacaram para o F3 as variáveis: Fes, Mi e COT (15,57 \%), enquanto o F4 variáveis Fes e Feo (13,24 $\%)$ foram mais sensíveis. 
A argila apresentou carga fatorial próximo a 1, sendo uma variável discriminante para o fator 4 , assim como o $\mathrm{Ca}^{2+} \mathrm{e} \mathrm{Na}^{+}$.

Figura 1 - Círculo de correlações Fator 1 x Fator 2 (A) e Plano fatorial Fator 1 x Fator 3 (B), apresentando a relação dos atributos do solo e os ambientes em estudo. . Fonte: Própria
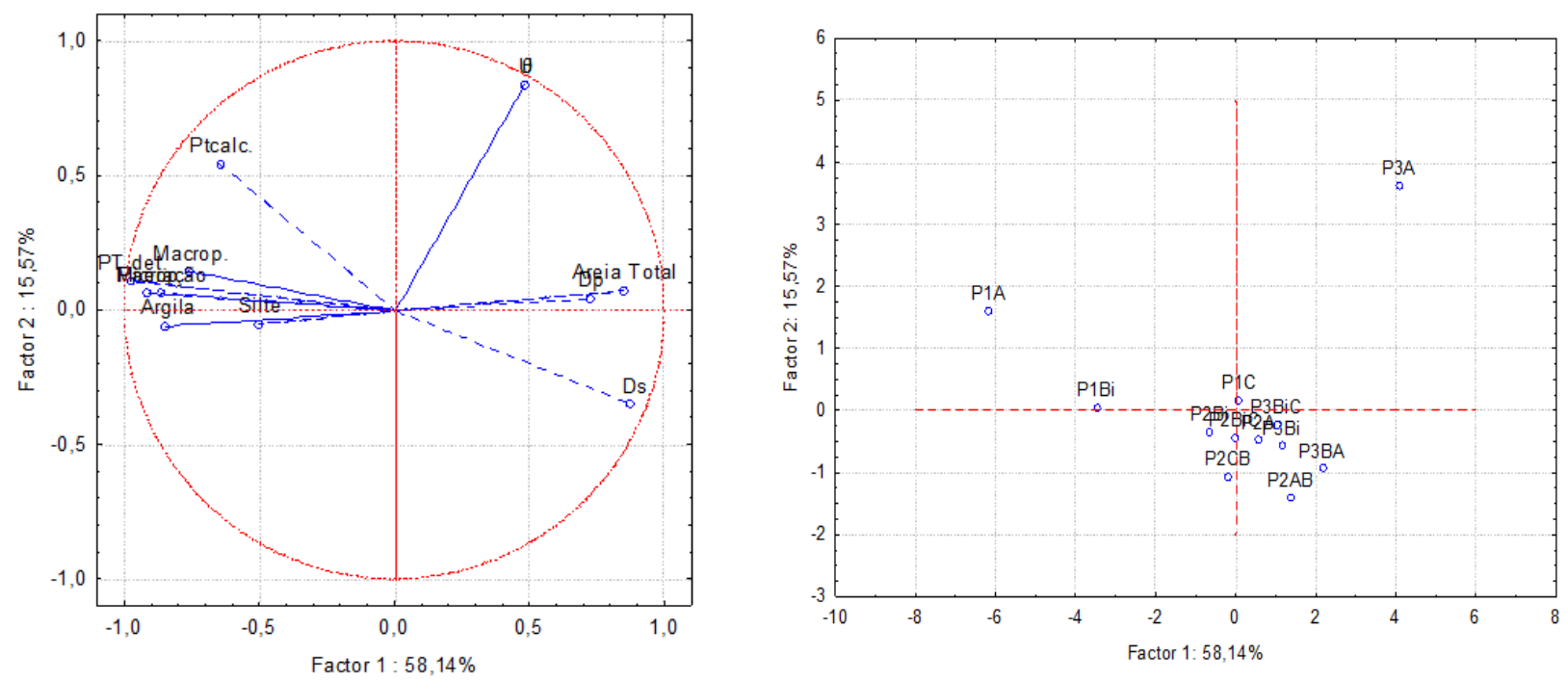

Figura 2-Círculo de correlações Fator 3 x Fator 4 (A) e Plano fatorial Fator 3 x Fator 4 (B), apresentando a relação dos atributos do solo e os ambientes em estudo. Fonte: Própria
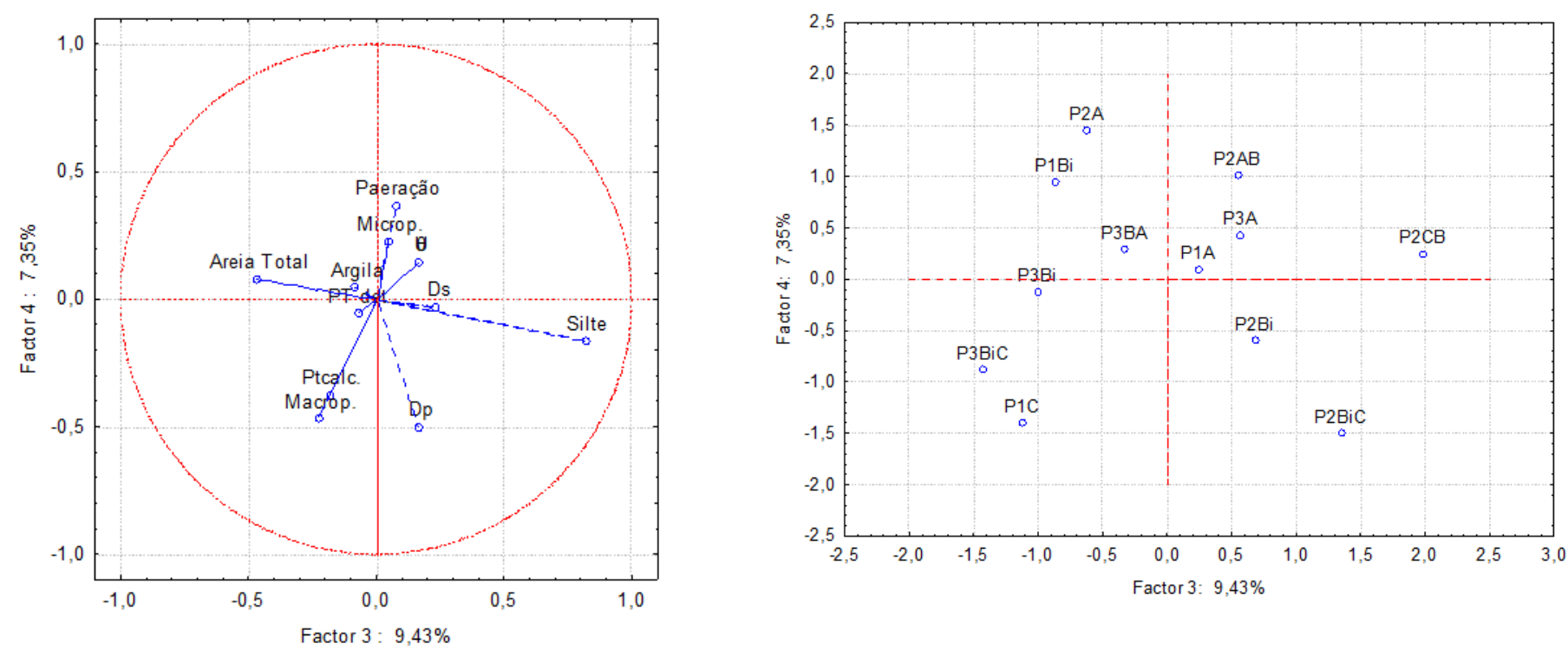


\section{Conclusões}

As classes de solos estudadas apresentaram de forma geral valores menores que 1 para razão de Ferro na forma amorfa e cristalina (Feo/Fed), condizendo com a natureza de ambiente mais intemperizados para os Latossolos e Argissolos, sendo os Cambissolos menos desenvolvidos, por apresentarem valores superiores da razão de Ferro.

As variáveis discriminantes de ferro amorfo (Feo) foram para o Latossolo Vermelhoamarelo em função da presença de maior teor de ferro na fração argila.

A fração argila apresentou inter-relação com os atributos químicos $\mathrm{Na}^{+} \mathrm{e} \mathrm{Ca}^{2+}$ na distinção das classes estudadas e o silte para a classe Cambissolo Háplico.

\section{Referências}

CASTILHO, K. B., CORTEZ, J. W., OLSZEVSKI, N., SALVIANO, A. M., \& TRINDADE, M. H. Análise multivariada da qualidade química de um Latossolo sob sistemas de manejo do solo. Agrarian, v. 10 n, 36. 162-169, 2017.

KÄMPF, N.; KLANT, E.; SCHNEIDER, P. Óxidos de ferro em Latossolos do Brasil Sudeste e Sul. Reunião de classificação, correlação de solos e interpretação de aptidão agrícola, v. 3, p. 153-183, 1988.

KAMPF, N.; DICK, D. P. Óxidos de ferro em Cambissolos brunos no Rio Grande do Sul e Sul de Minas Gerais. Revista brasileira de ciencia do solo. Viçosa. Vol. 8 (1984), p. 183$188,1984$.

MEHRA, O. P.; JACKSON, M. L. Iron oxide removal from soils and clays by a dithionitecitrate system buffered with sodium bicarbonate. In: Clays and clay minerals: proceedings of the Seventh National Conference. p. 317-327. 1960.

PÖTTER, R. O.; KÄMPF, N. Argilo-minerais e óxidos de ferro em Cambissolos e Latossolos sob regime climático térmico údico no Rio Grande do Sul. Revista Brasileira de Ciência do Solo, v. 5, n. 3, p. 153-159, 1981.

STASOFT.Statistica Version 7.0. StatSoft. Retrieved from: http://www.statsoft.com. 2004.

TEIXEIRA, P. C.; DONAGEMMA G. K.; WENCESLAU, A. F.; TEIXEIRA, G. Manual de Métodos de Análise de Solo. 3. ed. Rio de Janeiro: Embrapa Solos, 573 p. 2017.

VETTORI, L. Métodos de análise de solo. Equipe de Pedologia e Fertilidade do Solo, Ministério da Agricultura, 1969.

VICINI, L.; SOUZA, A. M. Análise multivariada da teoria à prática. Santa Maria: UFSM, CCNE, 2005. 